\title{
Estudo da relação entre início da atividade sexual e gravidez
}

\section{Study of relationship between early sexual activity and pregnancy}

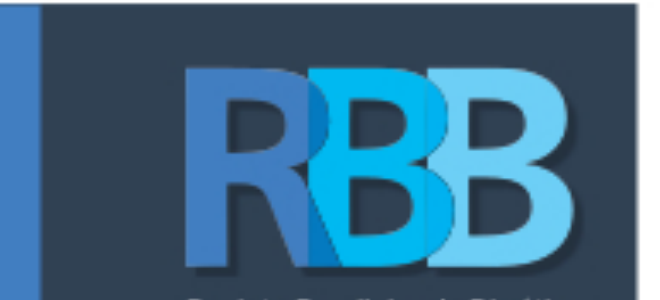

Revista Brasileira de Bioética

Mário Antônio Sanches

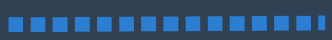

Pontifícia Universidade Católica do

Paraná, Curitiba, PR, Brasil

m.sanches@pucpr.br

Daiane Priscila Simão Silva

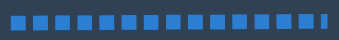

Pontifícia Universidade Católica do

Paraná, Curitiba, PR, Brasil

dpscientist@gmail.com

Shanny Mara Neves

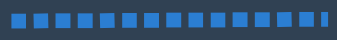

Pontifícia Universidade Católica do

Paraná, Curitiba, PR, Brasil

shannymara@hotmail.com

Castorina H. V. Casagrande

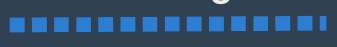

Pontifícia Universidade Católica do

Paraná, Curitiba, PR, Brasil castorina_vidal@hotmail.com

Thaiz Malakoski

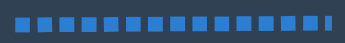

Faculdades Pequeno Príncipe,

Curitiba, PR, Brasil

thaizmalakoski@yahoo.com.br
Resumo: O objetivo deste artigo é abordar a temática da relação entre sexualidade e gravidez na adolescência, no contexto do planejamento familiar, a partir de revisão bibliográfica, e de uma pesquisa de campo realizada em localidades do Estado do Paraná que - situado no âmbito interdisciplinar da Bioética - permite análise de algumas causas da ausência ou presença do planejamento da parentalidade em pessoas que já tiveram filhos. Este artigo, focando na temática da sexualidade na adolescência é, portanto, resultado parcial desta pesquisa. O trabalho foi desenvolvido por meio de uma pesquisa de campo com 655 pessoas nos municípios de Curitiba e Jacarezinho no Paraná, onde foi aplicado o Instrumento de Pesquisa Planejamento da Parentalidade no Contexto da Bioética, validado pelo Grupo de Pesquisa. A literatura relacionada à sexualidade vem demonstrando uma tendência à diminuição de idade no tocante ao início da atividade sexual. A pesquisa realizada aponta para a mesma tendência e evidencia o ritmo com que isto ocorreu nas últimas décadas. A mesma situação se verifica em relação à gravidez na adolescência, pois tanto a revisão bibliográfica quanto a pesquisa de campo apontam para o aumento da gravidez na adolescência nas últimas décadas. Esta relação entre precocidade da atividade sexual e gravidez na adolescência é vista, no âmbito de nossa pesquisa, como um sintoma que releva precariedades no planejamento familiar.

Palavras-chave: adolescência, sexualidade, gravidez, bioética.

Abstract: This article discusses the relationship between sexuality and teen pregnancy in the context of family planning. It is a literature review and field research carried out in localities of the State of Paraná, Brazil. The research, situated within the interdisciplinary field of bioethics, allows analysis of some causes of absence or presence of family planning in people who have already had children. This article, focusing on sexuality in adolescence, is therefore a partial result of this research. The objective of this study was to map the presence or absence of parenting project in people who have had children in their teens, compared with the beginning of sexual activity of the research's participants. The study was developed by a field research with 655 persons in the cities of Curitiba and Jacarezinho, State of Parana, where the instrument applied was Parenting Planning in the Context of Bioethics, validated by the research group. The literature related to sexuality has been demonstrating a tendency to decrease in age for the starting of sexual activity. The survey points to the same trend and shows the pace with which this occurred in recent decades. The same situation applies to the teen pregnancy, since both the literature review and field research point to the rise in teenage pregnancy over the past decades. This relationship between precocity of sexual activity and teen pregnancy is seen, in our point of view, as a symptom of lack of family planning.

Keywords: adolescence, sexuality, pregnancy, bioethics.

Parecer de aprovação ética \# 409.841 pelo Sistema CEP/Conep 
A adolescência é um período de transição entre uma vida dependente e outra independente, afetada por transformações físicas, psíquicas e sociais. Dentro desse quadro, em meio a descobertas do próprio corpo e do relacionamento afetivo, pode ocorrer o início da atividade sexual que, num contexto de planejamento familiar precário, pode levar a uma gravidez na adolescência.

Existem várias definições de adolescência sob diferentes enfoques. Três delas, em um aspecto cronológico, são a da Organização Mundial da Saúde (OMS), que a caracteriza como o período compreendido entre 10 e 19 anos; o Estatuto da Criança e do Adolescente (ECA), regido pela Lei 8069, de julho de 1990, que considera esse período entre 12 e 18 anos de idade; e o Código Civil Brasileiro, que determina que a pessoa atinge a maioridade, aos 18 anos, aspecto que vem sendo questionado pela sociedade brasileira atualmente.

Sob uma caracterização psicossocial, adolescência é uma fase de transição, de um estado de dependência, para outro de relativa autonomia, em que o jovem sofre transformações biológicas, psíquicas e sociais (Moreira, Queiroz, Jorge, 2008), além de emocionais, que podem implicar numa vulnerabilidade individual e social (Gallo, 2011). Este conjunto de fatores determinará a identidade adulta, a autoestima e as relações afetivas (Silva, Tonete, 2006).

Na adolescência verifica-se uma mudança especial no jovem, sua maturação sexual. Há um consenso no mundo ocidental de que os indícios da puberdade, como eclosão hormonal e mudanças corporais, marcam o início da adolescência. Nesse contexto enquadra-se uma tendência global do início precoce das relações sexuais (Taquette, 2010), (Moreira, et al., 2013) pois "as transformações na vida sociocultural nas últimas décadas têm como uma de suas consequências o início da vida sexual de adolescentes cada vez mais cedo" (Brasil, 2006, p.14).

Segundo Brandão e Heilborn (2006, p.1425), na atualidade, "o exercício da sexualidade na adolescência torna-se uma via privilegiada para aquisição gradativa de liberdade e autonomia, mesmo sob o teto parental". A sexualidade, na reflexão atual, assume amplas dimensões. Algumas delas bastante desvinculadas da questão da reprodução, de modo que sexualidade e reprodução não estão necessariamente vinculadas (Valdivia, 2007).

Contudo, no período da adolescência, considerando a vulnerabilidade individual e social que estão expostos, a sexualidade e reprodução precisam ser estudadas nas suas inter-relações, principalmente num contexto do planejamento familiar. Desta forma, este artigo aborda a temática da relação entre sexualidade e gravidez na 
adolescência a partir de uma revisão bibliográfica e dos dados de uma pesquisa de campo realizada em cidades do Paraná.

\section{Metodologia}

\section{Pesquisa de Campo}

A pesquisa de campo consistiu da aplicação do Instrumento de Pesquisa Planejamento da Parentalidade no Contexto da Bioética, (SANCHES, et al. 2015) que consta de 53 questões relacionadas ao planejamento familiar. Foram consideradas para o presente trabalho as questões: "idade atual dos entrevistados"; "idade que iniciou um relacionamento sexual" e "idade da primeira gravidez / engravidou alguém pela primeira vez".

As entrevistas foram realizadas no ano de 2014, nos municípios de Curitiba e Jacarezinho, no Estado do Paraná. O projeto foi submetido e aprovado pelo Comitê de Ética em Pesquisa da PUCPR com parecer de número 409.841 na data de 25/09/2013. A abordagem dos sujeitos da pesquisa ocorreu em eventos educacionais, religiosos ou sociais organizados em comunidades da lgreja Católica. Portanto, o perfil dos entrevistados se caracteriza por serem agentes da lgreja, e que tenham ao menos 3 anos de participação ativa em alguma atividade eclesial. Os critérios de inclusão consistiram na idade superior a 18 anos, e já ter tido ao menos um filho.

\section{Análises Estatísticas}

As comparações entre as diferentes faixas etárias foram realizadas através do teste de Qui-quadrado (X2) da Independência. A correlação entre a "idade que iniciou um relacionamento sexual" e "idade da primeira gravidez / engravidou alguém pela primeira vez" foi avaliada através do coeficiente de correlação de Pearson para dados não paramétricos. Para todas as análises foi utilizado o programa IBM SPSS Statistics 20. Um nível de 5\% de significância foi adotado para todas as análises estatísticas realizadas.

\section{Resultados}

Foram entrevistadas 655 pessoas de diferentes faixas etárias Tabela 1. Dentre os entrevistados, 43,7\% tem acima de 46 anos, o que permite avaliar a relação entre a idade de início de relacionamento sexual e gravidez nas últimas cinco décadas de forma longitudinal. 
Tabela 1 - Perfil geral dos entrevistados

\begin{tabular}{r|c|c} 
Gênero & Percentagem & No de entrevistados \\
\hline Masculino & 0,27 & 176 \\
Feminino & 0,72 & 468 \\
18 e 25 & Intervalo de idade (anos) & 14 \\
26 e 35 & 0,02 & 104 \\
36 e 45 & 0,16 & 232 \\
46 e 60 & 0,35 & 218 \\
Acima de 60 & 0,33 & 68 \\
\hline
\end{tabular}

Fonte: Grupo de Parentalidade 2016

Nota: As percentagens e os entrevistados que faltam na tabela correspondem aqueles que não responderam as questões,

Considerando a idade atual dos entrevistados foi possível verificar que há uma diferença significativa na idade de início de um relacionamento sexual (Gráfico 1). As únicas faixas etárias que não diferiram foram entre 26 e 35 anos e entre 36 e 45 anos de idade $(X 2=3,27 ; p=0,51)$. Os grupos com maior diferença foram entre 18 e 25 anos e 46 e 60 e acima de 60 anos $(X 2=24,21 ; p=0,00)$. São 64,2\% dos participantes com idade atual entre 18 e 25 anos que iniciaram atividade sexual antes dos 18 anos; este índice cai para 47\% para os que se situam atualmente numa faixa etária entre 26 e 45 anos e vai para 28,9\% para os que se situam entre 46 e 60 anos e praticamente mantêm a mesma percentagem (27,9\%) entre os participantes da pesquisa que estão acima de 60 anos. Para estes grupos a diferença consiste em que a primeira relação ocorreu principalmente entre os 22 e 25 anos para quem tem acima de 60 anos (42,2\%) enquanto para a faixa etária entre 46 e 60 anos foi mais cedo, entre 19 e 21 anos para 44, 2\% dos entrevistados. 
Gráfico 1. Relação entre os anos de nascimento dos entrevistados e a idade em que iniciaram um relacionamento sexual.

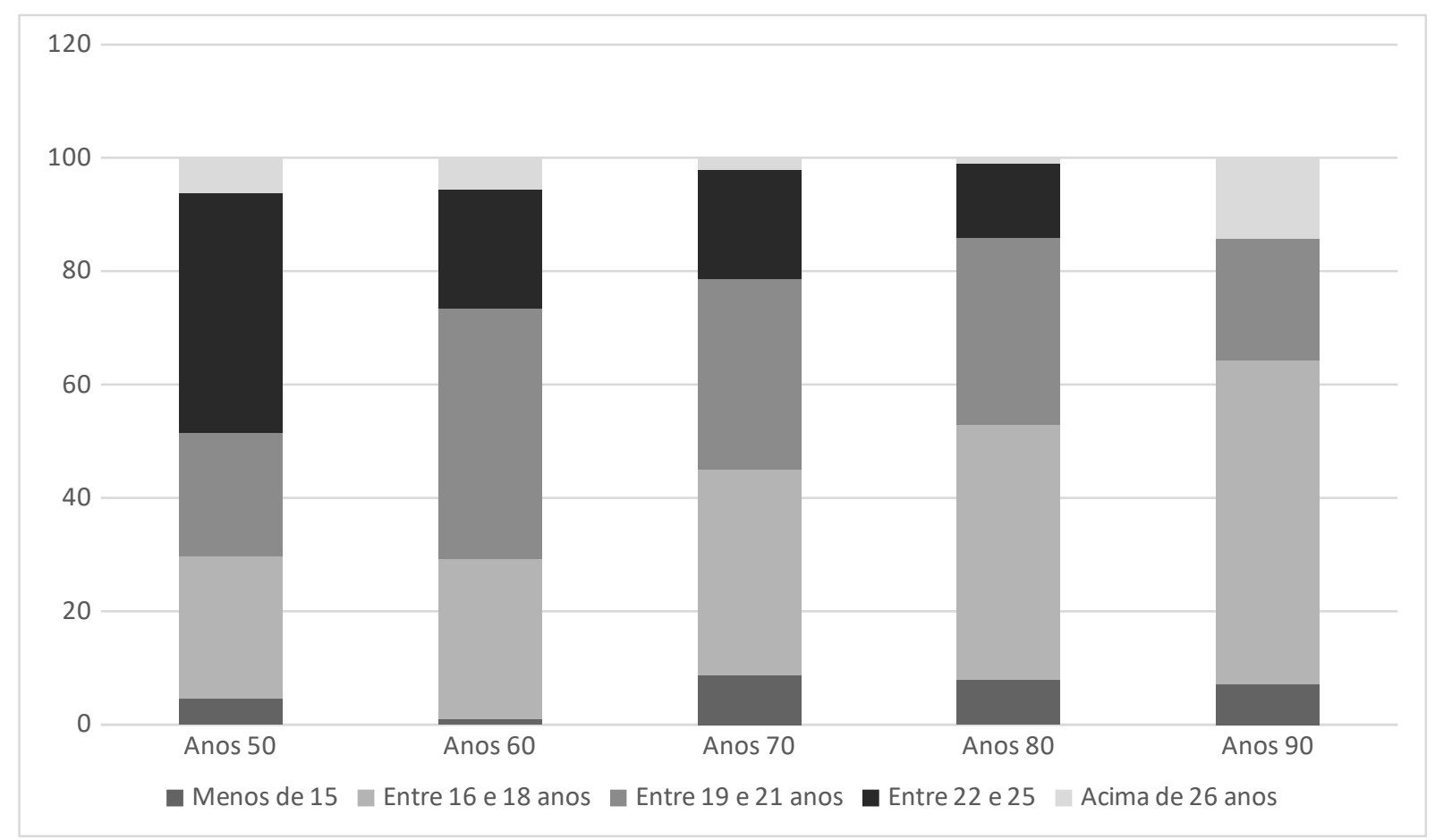

Fonte: Grupo de Parentalidade 2016

Entre faixas etárias avaliadas, não houve diferença significativa entre a idade da primeira gravidez/ engravidou alguém pela primeira vez, entre os grupos entre 18 e 25 anos e 26 - 35 anos (X2 = 2,18; $p=0,70)$, dessa forma, optou-se por agrupá-los. 0 grupo entre 18 e 35 anos de idade diferiu significativamente da faixa etária de 36 - 45 anos $(X 2=14,32 ; P=0,00)$ assim como o grupo entre $46-60$ anos $(X 2=21,06 ; P=$ 0,00). Como pode ser observado no gráfico 2, a diferença entre os grupos se dá pela diminuição da primeira gravidez entre os 22 e 30 anos de idade ao longo dos anos, e um aumento no número de gravidez na adolescência (faixa entre 16 e 18 anos). Ocorre também um acréscimo no número de gravidez acima dos 31 anos.

Houve correlação positiva (moderada) entre a idade do primeiro relacionamento sexual e a idade da primeira gravidez $(=0,40 ; p=0,00)$. 
Gráfico 2. Relação entre a idade atual dos entrevistados e a idade da primeira gravidez/ engravidou alguém pela primeira vez.

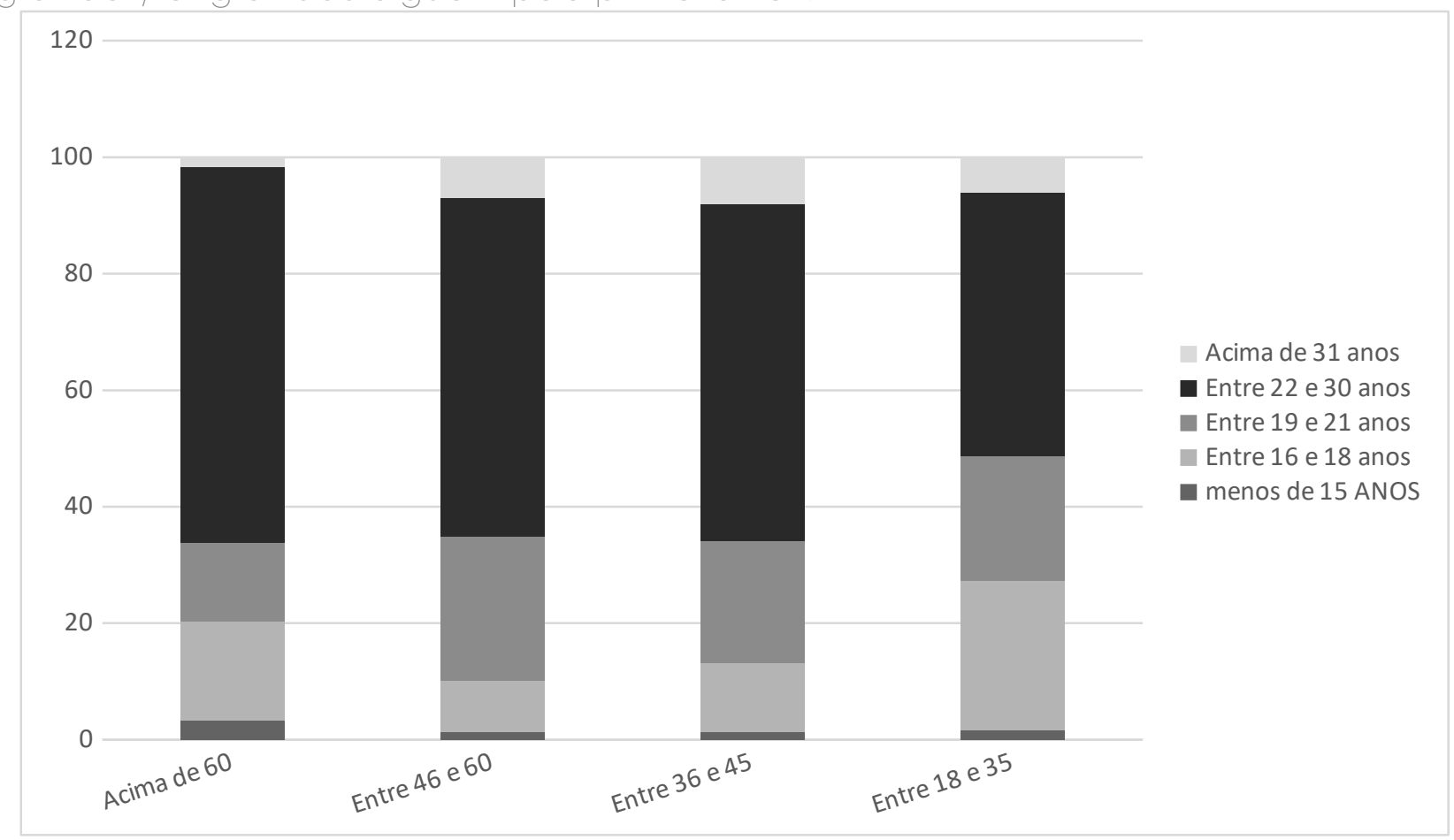

Fonte: Grupo de Parentalidade 2016

\section{Discussão}

Os resultados da pesquisa estão de acordo com os dados do Ministério da Saúde de 1998, onde 61\% dos jovens brasileiros entre 16 e 19 anos já haviam tido relações sexuais, sendo que para 40,2\% deles a primeira relação ocorreu antes dos 15 anos de idade (Brasil, 2000). Os valores encontrados estão um pouco acima dos resultados do Fundo das Nações Unidas para a Infância (Unicef), em 2002, com 32,8\% dos brasileiros entre 12 e 17 anos já tendo iniciado a atividade sexual (Brasil, 2006). Não foi possível identificar seguramente os valores para a faixa etária das últimas gerações, contudo, um estudo realizado com 1015 jovens em Porto Alegre, identificou que a primeira experiência sexual para mais da metade dos adolescentes (55,4\%) ocorreu antes dos 15 anos de idade (Cerqueira-Santos et al., 2010). Em uma maternidade de Ribeirão Preto, 47,1\% das jovens entrevistadas, iniciaram a atividade sexual entre 14 e 15 anos de idade e 37,1\%, entre 16 e 18 anos de idade. Constam ainda que a faixa etária mais frequente para o início das relações sexuais está entre 15 ou 16 anos, compreendem-se que esta precocidade se dá pelo estilo de vida moderno e influências de fatores ambientai (Almeida, Da Trindade, Gomes, 2003) (Berlofi et al., 2006). 
Inúmeros fatores podem levar o jovem a iniciar sua vida sexual precoce, como o ambiente em que estão inseridos, falta de lazer, exemplos familiares, o estilo de vida moderno, questões afetivas, como demonstração de amor pelo parceiro ou à própria necessidade de afirmação pessoal (Godinho et al., 2000), (Berlofi et al., 2006), numa etapa da vida em que há conflitos na formação de sua personalidade. Insere-se neste contexto a influência ativa da mídia atual, que faz apelos diariamente ao sexo, através de programas, novelas, propagandas entre outros (Moreira, Queiroz, Jorge, 2008).

No Brasil, cerca de um milhão de adolescentes dão à luz a cada ano (Silva, Tonete, 2008), e em 2011, mais de 500 mil partos foram de adolescentes entre 15 e 19 anos (Brasil, 2014). Os dados da pesquisa permitem identificar uma correlação positiva, ao longo dos anos, entre a precocidade do início das relações sexuais e a ocorrência da primeira gestação na adolescência. Segundo Schor (1995), quanto antes um adolescente inicia a vida sexual, menor é a chance de uso de algum método anticoncepcional e, por consequência, aumenta a probabilidade de gravidez logo nas primeiras relações.

Em algumas sociedades o início precoce das atividades sexuais não está relacionado com aumento da gravidez na adolescência e quando isto ocorre há sem dúvida o indicativo de um planejamento familiar precário. A taxa de fecundidade para a população adolescente é especialmente maior quando se trata de países mais pobres (Berlofi et al., 2006), (Cerqueira-Santos et al., 2010). A idade média da menarca vem decrescendo com o decorrer dos anos (Berlofi et al., 2012) o que, somado ao início precoce da vida sexual ativa, acentua a possibilidade de ocorrer gestação mais cedo na vida da jovem. Devido a essas questões pode-se afirmar que há um aumento na taxa de fecundidade para a população adolescente, especialmente quando se trata de países mais pobres (Berlofi et al., 2012) (Cerqueira-Santos et al., 2010).

Todo jovem tem direito à informação, à educação sexual e reprodutiva e ao sexo seguro (Brasil, b, 2006). Existem campanhas nacionais para prevenir a gravidez e a transmissão de Doenças Sexualmente Transmissíveis (DSTS). Disponibilizar esse tipo de orientação e oferecer opções de escolha ao jovem é uma boa medida de prevenção de uma gravidez não planejada (Berlofi et al., 2006).

Estudos mostram que muitas gestantes adolescentes têm conhecimento a respeito dos métodos de anticoncepção, porém, o impacto desse conhecimento em seu cotidiano ainda não é satisfatório. Exemplo disso é um estudo realizado por Guimarães e Witter (2007, p. 172), cujos resultados afirmam que 59\% das adolescentes de postos de saúde contidos no estudo, já haviam recebido informações sobre anticoncepção de algum profissional de saúde, porém, 63,6\% não faziam uso de nenhum método correspondente. 
grande desafio a ser superado pelo serviço público é a falta de informação sobre sexualidade e maternidade ou a existência de informações não consistentes entre muitos jovens. A qualidade da informação que chega até os mesmos é questionável, pois ainda existem dúvidas importantes entre eles (De Moura, 2011). Em muitos casos os jovens não conseguem traduzir a informação que dispõem em mudanças de comportamento (De Moura, 2011).

Os pais não transmitem orientação adequadamente por não conhecê-la ou por constrangimento (Moreira, Queiroz, Jorge, 2008). Existe também a possibilidade real de os pais acreditarem que negando esse tipo de informação aos filhos, estarão evitando o início precoce da vida sexual (Leal \& Leowenwall, 2005). A partir do momento em que houver superação da barreira de comunicação entre pais, adolescentes e profissionais de saúde, poderá haver uma diminuição efetiva dos casos de gestação indesejada na adolescência (Leal \& Leowenwall, 2005).

Conceitos sobre virgindade, casamento, maternidade e amor vem tendo valores modificados pela sociedade. Muitos adolescentes acabam assumindo papeis de adultos, porém sua maturidade psicológica não está necessariamente preparada para isso (Moreira, Queiroz, Jorge, 2008).

Frente a esse quadro, dados que afirmam que o número de adolescentes que engravidaram no Brasil aumentaram nos últimos anos, não surpreendem. O quadro das gestantes adolescentes é considerado, pelo Ministério da Saúde, de alto risco, do ponto de vista clínico, biológico e comportamental (Brasil,1999). A literatura afirma que este grupo está sujeito a complicações como eclampsia, anemia, parto prematuro e nascimento de bebês com baixo peso (Silva, Tonete, 2006). Além disso, pesquisas inerentes ao concepto dessas mães jovens, sugerem que eles podem apresentar maiores riscos de morbidade e mortalidade infantil, bem como atraso no seu desenvolvimento e problemas psicológicos (Vieira, 2006).

Entretanto, pior que uma gestação na adolescência é sua repetição, que pressupõe problemas como o pequeno intervalo interpartal ocasionando baixo peso nos recém-nascidos(...). Mulheres que iniciam a maternidade na adolescência tendem a ter um número maior de filhos(...) (Berlofi et al., 2006, p. 198)

Porém, os conflitos psicossociais associados à gravidez dessas jovens constituem-se mais marcantes em relação às alterações físicas (Leal \& Leowenwall, 2005). Esse evento repercute no âmbito psicológico, social e econômico dessas meninas (Silva, Tonete, 2006). Para Belo e Silva (2004), a gravidez na adolescência pode estar associada a diversos fatores, entre eles a violência doméstica, a baixos níveis socioeconômicos e de escolaridade, assim como a falta de perspectivas 
pessoais de vida. Socialmente os conceitos sobre virgindade, casamento, maternidade e amor vem sofrendo modificações e, dentro deste contexto, a gravidez na adolescência pode ocorrer como consequência dessa mudança de comportamento. A gravidez quando ocorre por falta de planejamento familiar, torna-se o centro de situações com fortes implicações para a gestante e a criança, para a família, e também para os centros de saúde. Dessa forma, o exercício da parentalidade acaba sendo 'imposto', sem que exista um projeto de parentalidade.

O início da vida sexual precoce entre os adolescentes se deve também as transformações ocorridas na sociedade nas últimas décadas (Brasil, 2006). Avaliando - perfil etário para gravidez/ou engravidou alguém, foi identificado que existe um aumento da primeira gestação nas faixas etárias de extremos, principalmente no limiar inferior, abaixo de 18 anos. Estes achados estão de acordo com dados do Instituto Brasileiro de Geografia e Estatística (IBGE) (Brasil, 2010) para os últimos 30 anos. Nesse contexto, há um impacto da idade da mulher em relação à qualidade da gestação, tendo as mulheres nos extremos de idade, em geral, resultados menos favoráveis que as mulheres entre 20 e 35 anos, chamadas de adultas jovens (Silva, Surita, 2009), período este que, foram encontrados um decréscimo no número de gestações.

No caso das adolescentes, a gravidez não planejada pode resultar, além das consequências obstétricas e para o concepto, em sobrecarga emocional, psíquica e social, repercutindo no desenvolvimento e no projeto de vida futura (Manfré, Queiroz, Mathes, 2010), e a maioria delas não está preparada para assumir esse desafio (Moreira, Queiroz, Jorge, 2008).

Estudos concordam, parte das adolescentes planejam a ocorrência da gravidez, uma vez que podem encontrar nesse acontecimento o reconhecimento social que almejam. Leal \& Leowenwall, (2005); Correia et al., (2011); Barreto (2011), afirmaram que mais da metade de sua população de estudo, atribuiu o próprio desejo de engravidar como única motivação para a gestação na adolescência.

O período da gestação é marcado por grandes transformações no organismo da mulher, que naturalmente requerem uma maior atenção afetiva para si. Esse fator, associado às alterações inerentes à própria fase da adolescência, acaba gerando insegurança, fragilidade e ansiedade na jovem (Moreira, Queiroz, Jorge, 2008).

Algumas pesquisas apontam que a maternidade pode adquirir um caráter de centralidade na vida dessas meninas, pois podem encontrar na gravidez uma possibilidade de reconhecimento social (Leal \& Leowenwall, 2005) (Correia et al., 2011). Esta pode ser uma questão que aflora, nas futuras mães, o desejo de engravidar. 
Apesar de todas as dificuldades encontradas, em um momento que gera expectativa, e turbulência, muitas mães adolescentes afirmam que desejaram a gravidez. Além disso, acreditam num futuro promissor para seus filhos (Leal \& Leowenwall, 2005). O estudo realizado em Porto Velho, Rondônia, afirma essa questão, foram entrevistadas 422 parturientes adolescentes, das quais 290 declararam que sua gravidez foi planejada (Gallo, 2011)

De acordo com Leal \& Leowenwall (2005) 48,1\% dos jovens, informaram ter recebido a notícia de sua gravidez com alegria, enquanto 22,1\%, com pouquíssima alegria, e 1,6\%, com ausência desse sentimento. Quanto ao modo como os entrevistados reagiram à gravidez é interessante notar que os dados revelam contradição na relação entre a percentagem dos que afirmam ter planejado $(3,1 \%)$ com a percentagem dos que receberam a notícia da gravidez com alegria $(48,1 \%)$. Por ser um tema sensível é bem provável que se produza uma resposta politicamente correta: 'meu filho foi planejado'. E talvez aqueles que receberam o anúncio da gravidez com alegria 48,1\%, de fato estavam na expectativa de um filho. É notável perceber que os entrevistados que moravam com os pais receberam o anúncio da gravidez com maior dificuldade e apenas 3\% expressaram que o anúncio foi alegre.

As meninas que optam pelo aborto encaram a mesma situação com outra perspectiva. No estudo realizado por (Chaves et al., 2010), no qual pesquisou-se jovens que tiveram sua gestação interrompida, verificou-se que 98\% delas, foi devido a uma causa voluntária. Entre 164 adolescentes consideradas que tiveram um aborto provocado, 77,4\% assumiram ter usado misoprostol.

Existem uma diversidade de posicionamento quando o assunto é aborto. Uma das correntes é referente ao direito da mulher de realizar esse ato sob cuidados no âmbito hospitalar, devido a questões definidas. A outra é daqueles que defendem unicamente o aborto legal e por anomalia fetal grave. A terceira corresponde a uma forte corrente que é contrária a qualquer tipo de aborto, fortalecida por ideias morais e religiosas (Vieira, 2006)

É necessária a ressalva de que, independentemente da decisão da adolescente, devem ser respeitados os princípios da não-maleficência e da beneficência, ou seja, ela tem total direito humano de receber os cuidados médicos, psicológicos e sociais necessários, sem sofrer qualquer tipo de mal, na circunstância em que ela se encontrar.

Ao se tratar dos aspectos socioeconômicos ligados a esse fenômeno, ainda correlaciona-se o maior número de ocorrências de gravidez na adolescência, no meio em que há maiores índices de pobreza e baixa escolaridade, apesar de poder 
acontecer em todos os meios sociais (Cerqueira-Santos, 2010), (De Moura, 2011). A exemplo disso, dados do estudo realizado na cidade de Botucatu-SP, informam que mais da metade das adolescentes grávidas entrevistadas, tinham uma renda familiar inferior a 3 salários mínimos, e a escolaridade das mesmas, era abaixo daquela esperada para sua idade (Godinho, Schelp, Parada, Bertoncello, 2000).

Essa situação resulta no declínio do padrão de vida e muitas vezes em desamparo social (Leal \& Leowenwall, 2005). A perpetuação da pobreza, quando inseridas nesse meio, é inevitável, uma vez que muitas adolescentes abandonam os estudos, possivelmente devido à vergonha de assumirem uma gestação frente a colegas e professores. Esse fato reflete nos índices de baixa escolaridade dessa população. Consequentemente ficam em desvantagem frente a um mercado de trabalho cada vez mais competitivo e exigente (Godinho, Schelp, Parada, Bertoncello, 2000) acarretando consequências para si, para seu filho e para a sociedade (Meinck et al. , 2011). Posteriormente, a pouca profissionalização, a baixa renda e a escolaridade defasada dessa mãe, irão interferir no futuro da criança que vai nascer (Vieira, 2006).

\section{Considerações Finais}

Os dados da pesquisa de campo, assim como a literatura relacionada à sexualidade, vêm demonstrando uma tendência à diminuição da idade no tocante ao início da atividade sexual. A mesma situação se verifica em relação à gravidez na adolescência, com aumento da gravidez na adolescência nas últimas décadas. Esta relação entre precocidade da atividade sexual e gravidez na adolescência é vista, no âmbito da pesquisa, como um sintoma que releva precariedades no planejamento familiar. O contexto em que a pesquisa fora realizada, com membros da lgreja Católica em localidades do estado do Paraná, Sul do Brasil, não revelou diferenças significativas entre a literatura da população Brasileira em geral, apesar da vasta diversidade social e das condições de culturais da juventude no País.

Desta forma, discutir a sexualidade na adolescência é olhar para o adolescente como sujeito susceptível do exercício da maternidade/paternidade de forma não planejada e no âmbito da reprodução humana, o projeto de ser pais - projeto de parentalidade - precisa ser situado no contexto do projeto de vida total das pessoas envolvidas e da vida do casal. $O$ desenvolvimento da sexualidade é um processo gradativo e intimamente relacionado com a personalidade e amadurecimento pessoal. Desvelar a amplitude da inter-relação entre sexualidade e o processo reprodutivo, se faz necessário em vista da vivência saudável da adolescência. Haja vista que sexualidade e reprodução não são apenas processos biológicos, mas globais no desenvolvimento humano, a discussão deve ir para além do uso de métodos 
contraceptivos ou da liberdade sexual. Devem estar inseridos num âmbito mais amplo de educação, saúde e vinculados às pautas de planejamento familiar.

\section{Referências}

1. Almeida, AM, Da Trindade, RFC, Gomes, FA. Maternidade na Adolescência: um Desafio a Ser Enfrentado. Rev Bras Enferm; 56(5): 519-522, set-out, Brasília, 2003

2. Barreto, MM et al. Representação Social da Gravidez na Adolescência para Adolescentes Grávidas. Rev Rene, 12(2):384-92, Fortaleza, 2011

3. Berlofi et al. Prevenção da Reincidência de Gravidez em Adolescentes: Efeitos de um Programa de Planejamento Familiar. Acta Paul Enferm; 19(2):196-200, 2006

4. Belo M, Silva JLP. Conhecimento, atitude e prática sobre métodos anticoncepcionais entre adolescentes gestantes. Rev Saúde Pública;38(4):479-87, 2004.

5. Brandão ER, Heilborn ML. Sexualidade e gravidez na adolescência entre jovens de camadas médias do Rio de janeiro. Caderno de Saúde Pública. 2006; 22(7):1421-30

6. Brasil. Ministério da Saúde. Secretaria de Atenção à Saúde. Departamento de Ações Programáticas Estratégicas. Direitos Sexuais, Direitos Reprodutivos e Métodos Anticoncepcionais. Brasília: Ministério da Saúde, 2006

7. _________ Ministério da Saúde - DATASUS [homepage internet] Informações de Saúde. Nascidos Vivos - Brasil. 2011 http://tabnet.datasus.gov.br/cgi/tabcgi.exe? sinasc/cnv/nvuf.def Acesso em 02 de fevereiro de 2014.

8. ________ Ministério da Saúde. Secretaria de Atenção à Saúde. Departamento de Ações Programáticas Estratégicas. Direitos Sexuais, Direitos Reprodutivos e Métodos Anticoncepcionais. Brasília: Ministério da Saúde, 2006.

9. ________ Saúde e Desenvolvimento da Juventude Brasileira: Construindo uma Agenda Nacional. Ministério da Saúde, Secretaria de Políticas de Saúde. Brasília, 1999.

10. __________ Ministério do Planejamento, Orçamento e Gestão. Instituto de Geografia e Estatística (IBGE). Diretoria de Pesquisas. Coordenação de População e Indicadores Sociais. Síntese de Indicadores Sociais: uma Análise das Condições de Vida da População Brasileira. Rio de Janeiro, 2010.

11. ________ Ministério da Saúde, Secretaria de Políticas da Saúde, Coordenação Nacional de DST/AIDS. Comportamento Sexual da População Brasileira e Percepções do HIV/AID. Brasília, 2000.

12. CERQUEIRA-SANTOS et al. Gravidez na Adolescência: Análise Contextual de Risco e Proteção. Psicologia em Estudo, v.15, n.1, p. 73-85, jan-mar, Maringá, 2010.

13. Correia, DS et al. Adolescentes Grávidas: Sinais, Sintomas, Intercorrências e Presença de Estresse. Rev Gaúcha Enferm; 32(1):40-7, mar, Porto Alegre, 2011

14. Chaves JH et al. Abortamento Provocado na Adolescência sob a Perspectiva Bioética. Rev Bras. Saude Matern Infant; 10 (Supl.2): 311-19, dez, Recife, 2010

15. De Moura LN. Informação sobre Contracepção e Sexualidade entre Adolescentes que Vivenciaram uma Gravidez. Acta Paul Enferm; 24(3): 320-26, 2011 
16. Gallo JH. Gravidez na Adolescência: a Idade materna, Consequências e Repercussões. Rev Bioética; 19(1): 79-95, 2011.

17. Godinho RA, Schelp JRB, Parada CMG., Bertoncello NMF. Adolescentes e Grávidas: Onde Buscam Apoio? Rev latino-am. Enfermagem, v.8, n.2, p. 25-32, abril, Ribeirão Preto, 2000.

18. Guimarães EA, Witter GP. Gravidez na Adolescência: Conhecimentos e Prevenção entre Jovens. Boletim Academia paulista de Psicologia - Ano XXVII, n. 2/07: 167-180, 2007.

19. Leal AC, Leowenwall M. Percepções da Gravidez para Adolescentes e Perspectivas de Vida Diante da Realidade Vivenciada. Cogitare Enferm; 10(3):44-52, set-dez, 2005.

20. Manfré CC, Queiróz SG, Matthe ÂCS. Considerações atuais sobre gravidez na adolescência. R. bras. Med. Fam. e Comum., Florianópolis, v. 5, n. 17, p. 48-54, jan./dez. 2010.

21. Meinck SMK et al. Perfil Socioeconômico e Demográfico de Puérperas Adolescentes. Cogitare Enferm; 16(3): 486-91, jul-set, 2011

22. Moreira RM et al. Adolescência e Sexualidade: Uma Reflexão com Enfoque Bioético. Adolesc Saude; 10 n. 3, 61-71, jul-set, 2013.

23. Moreira TMM, Viana DS, Queiroz MVO, Jorge MSB. Conflitos Vivenciados pelas Adolescentes com a Descoberta da Gravidez. Rev Esc Enferm USP; 42(2): 312-20, 2008.

24. Sanches MA, Krum JC, Sato, ES, Santos RB. Planejamento da parentalidade no contexto da bioética : busca de uma nova abordagem para pesquisa.. 1. ed. Curitiba: PUCPRess, 2015. v. 1. 301p .

25. Silva JLCP, Surita FGC. Idade materna: resultados perinatais e via de parto. Rev Bras Ginecol Obstet. 31(7):321-5, 2009.

26. Silva L, Tonete VLP. A Gravidez na Adolescência Sob a Perspectiva dos Familiares: Compartilhando Projetos de Vida e Cuidado. Rev Latino-am Enfermagem 14(2):199206, março-abril 2006.

27. Schor N. Adolescência e Anticoncepção: Conhecimento e Uso. Tese de Livre Docência, São Paulo: Faculdade de Saúde Pública, Universidade de São Paulo; 1995.

28. Taquette SR. Conduta Ética no Atendimento à saúde de Adolescentes. Rev Adolescência e Saúde; v. 7, n. 1, janeiro de 2010.

29. Valdivia VB. La violencia contra la mujer y los derechos sexuales y reproductivos. Revista Derecho PUCP, n. 61, p. 81-110, 2007.

30. Vieira TR. Bioética: Temas Atuais e Seus Aspectos Jurídicos. Editora Consulex. Brasília, 2006.

Recebido em: 25/05/2017. Aprovado em: 13/10/2017. 